\title{
Connection between Gravity and Electromagnetism
}

\author{
OCTAVIAN BALACI
}

tavib72@mail.com

\begin{abstract}
A new interpretation of electrodynamics and gravity is presented, based on the idea that the electromagnetic and gravitational properties of vacuum are connected. The space and time are treated as imaginary concepts. With this, the electrodynamics and gravitational phenomena can be explained with a Galilean invariant vacuum. Also a new way to explain the gravitational attraction will result.
\end{abstract}

\section{Introduction}

The electromagnetic field theory initiated by Faraday and Maxwell presume that a special medium called luminiferous aether is the bearer of electric and magnetic fields, aether which was considered at absolute rest in the entire universe. This model of aether and some variants of it were in contradiction with many observed phenomena involving the interpretation of electrodynamics in conditions of relative motion. All this problems open the way to Special Relativity which consider the electromagnetic field at rest relative to any inertial reference frame. This mean that the electromagnetic field and the quantum vacuum, which are physical entities, are arbitrary chosen in regard of their movement stat. But if a physical entity like the quantum vacuum background is arbitrary relative, then serious problems appear. This lead to a breaking of causality, an arbitrary change of the coordinate system will make the vacuum background to behave accordingly without a physical cause. Also, in this case, other problems exist, like the inability to explain the aberration of light in the frame of Special or General Relativity. If we 
take the observer located on earth as frame of reference, the space-time continuum and the electromagnetic field are at rest relative to the observer. As a consequence, no angle of aberration is predicted in the case of a source with a spherical emission (star), which is contradicted by well known astronomical observations. The result is different if we analyze the same problem, considering the star as frame of reference.

A different way to understand electrodynamics and gravity will be presented here, based on an assumed connection between the electromagnetic and gravitational properties of vacuum. In quantum mechanics the vacuum no longer can be considered pure empty space, but a quantum vacuum background which is considered Lorentz invariant. However, as we already point out, Lorentz invariance lead to serious errors. We will show here that the electrodynamics and gravitational phenomena can be correctly explained only with a Galilean invariant vacuum background, which not violate causality and will lead us to clear explanations of aberration of light and other phenomena. The space and time will be considered here as imaginary concepts. The electromagnetic field is immobile in the vacuum background, as it was in the luminiferous aether and the space-time continuum.

\section{Gravitational Vacuum.}

The most simple and direct explanation for the deflection of light by gravity, knowing that the light is an electromagnetic wave, can be done if we assume that the gravitational potential change the properties of vacuum, which will produce a gravitational refraction of light. This also will have many other implications like the change of the speed of light and a new way to explain the nature of gravitational attraction.

The vacuum is characterized by local properties: gravitational potential $(\Gamma)$, electric permittivity $\left(\varepsilon_{0}\right)$, magnetic permeability $\left(\mu_{0}\right)$. In absence of any ponderable matter, the vacuum properties will be homogeneous distributed in space, having everywhere the same positive value of gravitational potential, which is the vacuum background gravitational potential. Now if the ponderable matter, characterized by its heavy mass, is present in space, it will modify the local gravitational potential of vacuum in accordance with the Poisson equation of Newtonian gravity

$$
\nabla^{2} \Gamma=4 \pi G \rho_{m}
$$


where $\mathrm{G}$ gravitational constant, $\rho_{m}$ mass density. Assuming a spherical mass distribution $(M)$ we can express the gravitational potential at a distance $(R)$ from the center of mass and outside of it, as follow

$$
\Gamma=\Gamma_{0}-\frac{G M}{R}
$$

where $\Gamma_{0}$ background gravitational potential in the absence of mass. As we observe, the gravitational potential of vacuum decrease in the vicinity of masses and this will modify its electromagnetic properties. The electromagnetic properties of vacuum are not universal constants, but are dependent by the local gravitational potential. The electric permittivity of vacuum depend by the gravitational potential as follow

$$
\varepsilon_{0}=\kappa \cdot f_{1}(\Gamma)
$$

where $\kappa$ and $f_{1}$ are undetermined constant and function. The permittivity also must increase with the proximity to heavy masses, in order to explain the gravitational refraction of light and the gravitational force. The magnetic permeability must depend by the gravitational potential in a manner similar of permittivity. To explain this we consider an electromagnetic wave which propagate through the vicinity of a heavy cosmic body, the wave direction is deflected due to the variation of electromagnetic properties (gravitational refraction), but not suffer reflections, because such a phenomenon has not been observed. Consequently we can assume that the electromagnetic waves not suffer reflections when traversing zones with different vacuum properties, which can happen only if the vacuum impedance is independent by the gravitational potential.

$$
\begin{aligned}
Z_{0} & =\sqrt{\frac{\mu_{0}}{\varepsilon_{0}}} \\
\mu_{0}=Z_{0}^{2} \varepsilon_{0} & =Z_{0}^{2} \kappa \cdot f_{1}(\Gamma)
\end{aligned}
$$

As a result the electric permittivity and the magnetic permeability become higher and the propagation speed of electromagnetic field become lower in zones with lower gravitational potential. For determination of constant and function from (3) we will use the equality between the inertial and heavy mass, but first we must express the electromagnetic force due to permittivity variations. 


\subsection{Gravitational Force}

One result of spatial variation of electromagnetic properties, is the gravitational force. Let consider a small electric charge characterized only by its charge $q$ distributed on the surface of a sphere with radius $b$, at rest, sitting at a distance $R$ from a heavy mass $M$. The gravitational potential of vacuum decrease toward the center of mass as described by (2), consequently the electric permittivity increase. The electric field that surround the charge (unimportant if positive or negative) contain an amount of energy, if we consider the approximation that the variation of permittivity over the domain of integration cancel, we have the field energy

$$
U_{e 0}=\frac{q^{2}}{8 \pi \varepsilon_{0} b}
$$

The energy of electric field decrease when the charge approach the heavy mass due to increase of $\varepsilon_{0}$, the lost energy is converted into work of a force which is the gravitational attraction force. Because the neutral bodies are composed from atoms which have internal electric fields, result that they are attracted too, actually all bodies which have internal electromagnetic energy experience this attractive force in zones with spatial variable vacuum properties.

Because the permittivity is no longer equal around the outer area of sphere, the charged sphere surface no longer have a constant electric potential and tangential components of electric field appear. Supposing that the electrical charge can move freely on the spherical surface, this charge will redistribute over the surface until the surface electric potential will be constant, the electric field becoming again normal on surface. Now the charge density is no longer uniformly distributed over the surface of sphere, being denser where the permittivity is higher and the gravitational potential lower. Because the electric field is the same over the surface, a net attraction force appear, fueled by the decreasing electric field energy, representing the gravitational force.

When the charged sphere move to areas with a lower gravitational potential, the outside oriented electric forces on the surface of sphere will decrease and the inside oriented forces that hold the charged surface in equilibrium also must decrease, consequently the gravitational potential must have influence over the inside forces and over the radius of the charged sphere. In order to obtain the expected expression for the gravitational force, the charge radius 
(b) must increase with the decreasing of gravitational potential as follow

$$
b=\alpha \cdot f_{2}(\Gamma)
$$

where $\alpha$ and $f_{2}$ are the second undetermined constant and function. To resolve this we consider the charge at rest and make use of the observed fact that the inertial mass and the heavy mass are equal. Replacing the permittivity and radius in (6) result

$$
U_{e 0}=\frac{q^{2}}{8 \pi} \cdot \frac{1}{\kappa \alpha \cdot f_{1}(\Gamma) \cdot f_{2}(\Gamma)}
$$

The gravitational force must be

$$
\boldsymbol{F}_{\boldsymbol{g} \mathbf{0}}=-\frac{\mathrm{d} U_{e 0}}{\mathrm{~d} R} \cdot \mathbf{e}_{\mathbf{R}}=-m_{h 0} \cdot \frac{G M}{R^{2}} \cdot \mathbf{e}_{\mathbf{R}}
$$

where $\mathbf{e}_{\mathbf{R}}$ is the versor of $\boldsymbol{R}$, and $m_{h 0}$ is the rest heavy mass of charge $q$. The only way to satisfy (9) is

$$
f_{1}(\Gamma) \cdot f_{2}(\Gamma)=\frac{1}{\Gamma}
$$

with this will result

$$
\boldsymbol{F}_{\boldsymbol{g} \mathbf{0}}=-\frac{\mathrm{d} U_{e}}{\mathrm{~d} R} \cdot \mathbf{e}_{\mathbf{R}}=-\frac{q^{2}}{8 \pi \kappa \alpha} \cdot \frac{\mathrm{d} \Gamma}{\mathrm{d} R} \cdot \mathbf{e}_{\mathbf{R}}=-\frac{q^{2}}{8 \pi \kappa \alpha} \cdot \frac{G M}{R^{2}} \cdot \mathbf{e}_{\mathbf{R}}
$$

and the rest heavy mass

$$
m_{h 0}=\frac{q^{2}}{8 \pi \kappa \alpha}
$$

At rest the inertial mass depend only by the electric field energy and must be as follow

$$
m_{i 0}=\frac{U_{e 0}}{c^{2}}=\frac{\mu_{0} q^{2}}{8 \pi b}=\frac{q^{2}}{8 \pi} \cdot \frac{Z_{0}^{2} \kappa \cdot f_{1}(\Gamma)}{\alpha \cdot f_{2}(\Gamma)}
$$

To satisfy the equality $m_{i 0}=m_{h 0}$, one condition is that the inertial mass must be independent of the gravitational potential, this lead to $f_{1}(\Gamma)=f_{2}(\Gamma)$ and considering (10) result

$$
f_{1}(\Gamma)=f_{2}(\Gamma)=\frac{1}{\sqrt{\Gamma}}
$$


Also we have the equality

$$
\frac{q^{2} Z_{0}^{2} \kappa}{8 \pi \alpha}=\frac{q^{2}}{8 \pi \kappa \alpha}
$$

which lead to

$$
\kappa=\frac{1}{Z_{0}}
$$

The inertial and heavy mass at rest, become

$$
m_{0}=m_{i 0}=m_{h 0}=\frac{Z_{0} q^{2}}{8 \pi \alpha}
$$

Taking into account (16) and (14), we can write the permittivity, the permeability and the speed of light as follow

$$
\begin{aligned}
\varepsilon_{0} & =\frac{1}{Z_{0} \sqrt{\Gamma}} \\
\mu_{0} & =\frac{Z_{0}}{\sqrt{\Gamma}} \\
c & =\sqrt{\Gamma}
\end{aligned}
$$

From the equation (20) result that the local gravitational potential equal the square of the local speed of light.

Above was expressed the electrical part of the gravitational force at rest. At near to rest approximation the magnetic field energy alone have no contribution to the gravitational forces. To account for this we can express the magnetic field energy of a moving charged sphere relative to the local vacuum with a low velocity. The magnetic field energy produced by this movement is

$$
U_{m 0}=\frac{4}{3} \cdot \frac{\mu_{0} q^{2} v^{2}}{16 \pi b}=\frac{4}{3} \cdot \frac{Z_{0} q^{2} v^{2}}{16 \pi \alpha}
$$

which is independent by the gravitational potential. However we used here near to rest approximation for simplicity, we will show later that, when the propagation effects are taken into account, the gravitational force, the heavy and inertial mass are the effect of the total electromagnetic energy.

From the previously description, result that the gravitational force is not powered by some gravitational field energy, which not exist, but by the body internal electromagnetic energy. The body internal electromagnetic energy also contribute to the phenomenon of inertia. This lead to the possibility that the entire concept of mass may be of electromagnetic nature. 


\subsection{Atomic Radius Variation}

Because the permittivity change with the gravitational potential, this in turn will lead to the change of atomic radius and the dimensions of bodies. The radius of an electron orbit around the nucleus is given by the equilibrium between the nucleus attraction force and the centrifugal inertial force of electron. The quantum effects have an important influence at a such small scale. Let consider for simplicity the Bohr model of atom, the electron orbital radius is

$$
r_{n}=\frac{4 \pi \varepsilon_{0} \hbar^{2} n^{2}}{\mathbb{Z} e^{2} m_{e 0}}=\frac{4 \pi \hbar^{2} n^{2}}{\mathbb{Z} e^{2} m_{e 0}} \cdot \frac{1}{Z_{0} \sqrt{\Gamma}}
$$

where $\mathbb{Z}$ is the atomic number and $Z_{0}$ vacuum impedance. The $m_{e 0}$ is the rest mass of electron and is independent by the gravitational potential for low velocities compared with $c$. The radius of atom vary with the gravitational potential in a similar way as the radius of our sphere of charge considered previously. The atomic radius and dimensions of bodies increase in lower gravitational potentials.

\subsection{The Gravitational Entrainment of Vacuum}

One important problem related with the vacuum background is its movement state. In absence of any ponderable matter, the homogeneous distributed vacuum background does not have a movement state inside of it and a point of vacuum background is at rest relative to other point of it. The presence of ponderable matter will modify the gravitational properties of vacuum and this properties will follow the movement of ponderable matter. This imply two possibilities.

The first possibility assume that only the properties are entrained which require that the variations in the vacuum properties, which follow the ponderable matter position, propagate through it with a finite speed. This will produce a time lag in the action of gravitational force.

The second possibility assume that the vacuum background behave in such a way that the gravitational potential of it have no time variations, which eliminate the propagation and the time lag of the gravitational forces. This require that the vacuum background must follow the movement of the gravitational equipotential surfaces. This imply that the entrainment of vacuum is dependent by the local gradient of the gravitational potential. The

material derivative of the gravitational potential at one vacuum point moving 
with velocity $u$ relative to a mass, is

$$
\frac{\mathrm{D} \Gamma}{\mathrm{D} t}=\frac{\partial \Gamma}{\partial t}+\boldsymbol{u} \cdot \nabla \Gamma=0
$$

The condition of gravitational entrainment request that the material derivative is zero which imply that all variations in the gravitational potential, including those produced by the partial time derivative, viewed by the vacuum background are zero, the vacuum moving to compensate.

The vacuum entrainment is influenced by the direction and magnitude of the gravitational acceleration and by the movement of masses, higher the influence of a heavy body to the gravitational acceleration in one point, lower the movement of vacuum from that point relative to that body, higher the entrainment exerted by that body. Below we will analyze two simple cases of particular interest.

First is the case of a massive body (like a star or planet) far away from other massive bodies, this body will impose the value of gravitational acceleration in its vicinity, other bodies having only negligible influence because of their very low masses or very large distances. In this case the vacuum from the near zone of the massive body (local vacuum), with a negligible error, is total entrained by this massive body. Small objects with negligible influence over the gravitational acceleration, moving in the proximity of it, will experience a "gravitational wind" due to their own movement relative to the massive body.

The second case is that of rotation of a massive body around an axis of symmetry. In this case the rotational movement produce no modification over the gravitational potential in its proximity (if we neglect the centripetal acceleration), consequently the vacuum will remain unaffected. As a result, this body itself and any object that rotate with it experience a "gravitational wind" due to the body rotation around its own axis. However the rotation is an accelerated motion and because of this the vacuum around the rotating body will suffer some influences due to this acceleration.

The consequence of vacuum gravitational entrainment over the electromagnetic field, which is immobile relative to vacuum, consist in the advection of the field by the vacuum movement, leading to the induction of new field components. 


\section{The Electromagnetic Field and Vacuum}

The electric and magnetic fields are immobile relative to the vacuum background, consequently sharing its movement state. The vacuum is used here as physical entity, relative to which are expressed all velocities which appear in the electrodynamics equations. We will use the Maxwell-Heaviside equations of electrodynamics, not only because are well known, but also because are valid in the actual conditions where the permittivity and permeability are no longer uniformly distributed in space.

The electrical charge $q$ with the volume density $\rho_{v}$ may move relative to vacuum with velocity $v$, which form the current density $\boldsymbol{j}=\rho_{v} \boldsymbol{v}$. Always a charge movement through vacuum imply that its electric field will suffer an advection with velocity $-v$. Similarly, if a magnet move relative to vacuum with velocity $v_{m}$, its magnetic field will suffer an advection with velocity $-v_{m}$. A zone of vacuum may move relative to another zone of vacuum (due to gravitational entrainment) with velocity $u$, this also produce field advection between the two zones. Let consider the electric and magnetic field vectors $E, D$ and $H, B$, additional medium (like substantial bodies) polarization $P$ and magnetization $M$. We have the relations

$$
\boldsymbol{D}=\varepsilon_{0} \boldsymbol{E}+\boldsymbol{P}
$$

and

$$
\boldsymbol{B}=\mu_{0}(\boldsymbol{H}+\boldsymbol{M})
$$

Let consider the spatial elements: volume element $\mathrm{d} V$, surface element $\boldsymbol{d} \boldsymbol{S}=$ $\mathbf{n d} S$ and line element $\boldsymbol{d} \boldsymbol{r}$, which are tied with the field frame which is the vacuum. The integral form of electric and magnetic flux equations are

$$
\oiint \boldsymbol{D} \cdot \boldsymbol{d} \boldsymbol{S}=q
$$

and

$$
\oiint \boldsymbol{B} \cdot \boldsymbol{d} \boldsymbol{S}=0
$$

where $q$ is the charge enclosed inside the surface of integration. Using the divergence theorem these two equations can be transformed into their local form

$$
\nabla \cdot \boldsymbol{D}=\rho_{v}
$$


and

$$
\nabla \cdot \boldsymbol{B}=0
$$

The induction equations in integral form are

$$
\oint \boldsymbol{E} \cdot \boldsymbol{d r}=-\frac{\mathrm{d} \Phi}{\mathrm{d} t}
$$

and

$$
\oint \boldsymbol{H} \cdot \boldsymbol{d} \boldsymbol{r}=I+\frac{\mathrm{d} \Psi}{\mathrm{d} t}
$$

where $I, \Phi$ and $\Psi$ are the charge current relative to vacuum, the magnetic and electric flux, encircled by the curve of integration. To obtain the local form of induction equations, first we must evaluate the total time derivative of the electric and magnetic flux and interpret it in relation with the vacuum. Because the induced field may be localized in a different frame than the inductive field, the time derivative of electric and magnetic flux, viewed by the induced frame, is equal with the flux of material derivative of inductive field. In this conditions, considering a generic vector $F$, the time derivative of its flux is

$$
\frac{\mathrm{d}}{\mathrm{d} t}\left(\iint \boldsymbol{F} \cdot \boldsymbol{d} \boldsymbol{S}\right)=\iint\left(\frac{\mathrm{D} \boldsymbol{F}}{\mathrm{D} t}\right) \boldsymbol{d} \boldsymbol{S}
$$

where

$$
\frac{\mathrm{D} \boldsymbol{F}}{\mathrm{D} t}=\frac{\partial \boldsymbol{F}}{\partial t}+(\boldsymbol{u} \cdot \nabla) \boldsymbol{F}=\frac{\partial \boldsymbol{F}}{\partial t}+\boldsymbol{u}(\nabla \cdot \boldsymbol{F})+\nabla \times(\boldsymbol{F} \times \boldsymbol{u})
$$

is the material derivative of the field $F$ viewed by the induced frame, $u$ is the velocity between the frame where the field is induced and the frame in which the inductive field $F$ is mathematically expressed. From physical point of view the induced frame is the vacuum, from mathematical point of view can be any frame. The inductive field can be mathematically expressed in the same frame as the induced field, when this velocity is zero, or the field can be expressed in another frame, when this velocity is nonzero. When the two frames (induced and inductive) correspond at two zones of vacuum in relative movement, we have a case of field advection effect. Using the Stokes theorem and taking into account the condition (29), the equation (30) can be expressed in local form as follow

$$
\nabla \times \boldsymbol{E}=-\frac{\partial \boldsymbol{B}}{\partial t}+\nabla \times(\boldsymbol{u} \times \boldsymbol{B})
$$


where $u$ is the velocity between the induced electric field frame and the inductive magnetic field frame. Similarly, but using condition (28), the equation (31) can be expressed in local form as follow

$$
\nabla \times \boldsymbol{H}=\rho_{v} \boldsymbol{v}+\frac{\partial \boldsymbol{D}}{\partial t}+\rho_{v} \boldsymbol{u}+\nabla \times(\boldsymbol{D} \times \boldsymbol{u})
$$

where $v$ is the velocity of charge relative to vacuum and $u$ is the velocity between the induced magnetic field frame and the inductive electric field frame. Regardless of how the field is mathematically expressed, the physical state of the field is always that of the frame of local vacuum. However is very common in the analysis of many electrodynamics problems to express the field in the frame of its source which may not coincide with the frame of vacuum, in these cases we must take into consideration the components containing the velocity $u$ from induction equations.

The electromagnetic force represent the exchange of electromagnetic energy, associated with a system, with the change of system position. In this case the electromagnetic force can be expressed as

$$
\boldsymbol{F}_{\text {em }}=-\frac{\mathrm{d} U_{e m}}{\mathrm{~d} r} \cdot \mathbf{e}_{\mathbf{r}}
$$

where $U_{e m}$ is the electromagnetic energy of the system and $r$ is the position of the system. Also the electromagnetic force can be expressed through the field-charge interaction. The force that act over a small electric charge $q$ in an electromagnetic field is

$$
\boldsymbol{F}_{\text {em }}=q \boldsymbol{E}+q \boldsymbol{v} \times \boldsymbol{B}
$$

where $v$ is the velocity between charge and vacuum, the magnetic field being immobile in vacuum. We have two components, one produced by the electric field and one produced by the presence of the magnetic field, also known as Lorentz force. However the Lorentz force appear only when the charge is in movement relative to vacuum. To clarify this type of problems, let consider the case of a charge and a magnet. If both the charge and the magnet are immobile relative to vacuum then we have no force acting on the charge. If the charge move relative to vacuum and magnet with velocity $v=v_{q m}$ then a Lorentz force act on the charge

$$
\boldsymbol{F}_{q m}=q \boldsymbol{v} \times \boldsymbol{B}=q \boldsymbol{v}_{q m} \times \boldsymbol{B}
$$


Now if the charge is immobile in vacuum and the magnet move relative to vacuum and charge with velocity $v_{m}=-v_{q m}$ then the magnetic field of the magnet suffer an advection with velocity $-v_{m}$ and an electric field is induced by this, the force on the charge being an electric force

$$
\boldsymbol{F}_{q m}=q \boldsymbol{E}=q \boldsymbol{v}_{q m} \times \boldsymbol{B}
$$

considering the magnetic field expressed in the frame of magnet. When both the charge and the magnet move relative to vacuum we have a resultant force composed from the two components described previously, the combined value of them is proportional with the velocity between charge and magnet. Because of this, one may wrong believe that the Lorentz force is given by the relative movement between charge and magnet, while in fact we have the sum of two forces.

The vacuum properties, permittivity and permeability, in the general case, are not uniformly distributed in space but form scalar fields. Because of this, the permittivity and permeability of vacuum are affected by differential operators, this imply that some methods (like electrodynamics potentials) where these parameters are treated as constants, are no longer general valid, except in the cases when these parameters can be approximate as constants. In the above field equations, the medium (including vacuum) parameters are inside the differential operators and represent the most general valid case, because are the direct consequence of experimental observations over the electromagnetic field.

\section{Electromagnetic Momentum.}

Let consider a small electric charge characterized only by its charge $q$ distributed on the surface of a sphere with radius $b$ (like in subsection 2.1), moving with constant velocity $v$ through vacuum in the condition of an uniformly distributed gravitational potential. This moving charge have a momentum of its electromagnetic field, with the volume density $\boldsymbol{D} \times \boldsymbol{B}$. The electric field around a hypothetical point charge in movement with constant velocity is affected by the finite speed of propagation (Lienard and Wiechert retarded 
potentials, or Heaviside and Thomson auxiliary system) and become

$$
\boldsymbol{E}_{\boldsymbol{v}}=\frac{q}{4 \pi \varepsilon_{0} r^{2}} \cdot \frac{1-\frac{v^{2}}{c^{2}}}{\left(1-\frac{v^{2}}{c^{2}} \sin ^{2} \theta\right)^{3 / 2}} \cdot \mathbf{e}_{\mathbf{r}}
$$

and the magnetic field

$$
\boldsymbol{B}_{\boldsymbol{v}}=\frac{1}{c^{2}}\left(\boldsymbol{v} \times \boldsymbol{E}_{\boldsymbol{v}}\right)
$$

where $\theta$ is the angle between the direction of movement and the direction of the position vector. The spherical charge at rest, in movement must change its shape until the tangential forces (electric and magnetic) at surface are canceled, which will happen when the charge take the form of an oblate spheroid on the direction of motion, with the longitudinal radius (on the direction of motion) becoming $\delta$ times shorter than the transversal radius which remain unaffected.

$$
\delta=\sqrt{1-\frac{v^{2}}{c^{2}}}=\sqrt{1-\beta}
$$

Now if we integrate the momentum density over the volume around the spheroidal shaped charge with radius

$$
b(\theta)=b \cdot \frac{\sqrt{1-\beta}}{\sqrt{1-\beta \sin ^{2} \theta}}
$$

we obtain the field momentum

$$
\begin{gathered}
\boldsymbol{P}_{\boldsymbol{e m}}=\int_{0}^{\pi} \int_{0}^{2 \pi} \int_{b(\theta)}^{\infty}\left(\boldsymbol{D}_{\boldsymbol{v}} \times \boldsymbol{B}_{\boldsymbol{v}}\right) r^{2} \sin \theta \mathrm{d} r \mathrm{~d} \varphi \mathrm{d} \theta \\
\boldsymbol{P}_{\text {em }}=\frac{q^{2} \boldsymbol{v}(1-\beta)^{3 / 2}}{c^{2} 8 \pi \varepsilon_{0} b} \cdot 2 \int_{0}^{\pi / 2} \frac{\sin ^{3} \theta}{\left(1-\beta \sin ^{2} \theta\right)^{5 / 2}} \mathrm{~d} \theta \\
\boldsymbol{P}_{\text {em }}=\frac{4}{3} \cdot \frac{q^{2} \boldsymbol{v}}{c^{2} 8 \pi \varepsilon_{0} b} \cdot \frac{1}{\sqrt{1-\beta}}
\end{gathered}
$$

in a similar way we obtain the electric field energy

$$
U_{e}=\frac{q^{2}(1-\beta)^{3 / 2}}{16 \pi \varepsilon_{0} b} \cdot 2 \int_{0}^{\pi / 2} \frac{\sin \theta}{\left(1-\beta \sin ^{2} \theta\right)^{5 / 2}} \mathrm{~d} \theta
$$




$$
U_{e}=\left(1-\frac{\beta}{3}\right) \frac{q^{2}}{8 \pi \varepsilon_{0} b} \cdot \frac{1}{\sqrt{1-\beta}}
$$

the magnetic field energy

$$
\begin{gathered}
U_{m}=\frac{v^{2}}{c^{2}} \cdot \frac{q^{2}(1-\beta)^{3 / 2}}{16 \pi \varepsilon_{0} b} \cdot 2 \int_{0}^{\pi / 2} \frac{\sin ^{3} \theta}{\left(1-\beta \sin ^{2} \theta\right)^{5 / 2}} \mathrm{~d} \theta \\
U_{m}=\frac{\beta}{3} \cdot \frac{q^{2}}{8 \pi \varepsilon_{0} b} \cdot \frac{1}{\sqrt{1-\beta}}
\end{gathered}
$$

the total energy

$$
U_{e}+U_{m}=\frac{q^{2}}{8 \pi \varepsilon_{0} b} \cdot \frac{1}{\sqrt{1-\beta}}
$$

We must make distinction between the momentum of field propagation and the momentum of charge movement relative to the local vacuum (inertial momentum), the field momentum being equal with the inertial momentum only in the case of free fields (electromagnetic wave fields) which form a balanced electromagnetic system. The fields created by the electric charge form an unbalanced system, additional stress (Poincare stress) is required to balance the system. Consequently we assume that when this stress is taken into account the $4 / 3$ factor must disappear. With this we can write the charge momentum relative to local vacuum as

$$
\boldsymbol{P}_{c \boldsymbol{v}}=\boldsymbol{P}_{e m}+\boldsymbol{P}_{s}=\frac{U_{e}+U_{m}}{c^{2}} \cdot \boldsymbol{v}
$$

The total charge momentum relative to distant vacuum is given by the charge momentum relative to local vacuum and an additional momentum produced by the gravitational entrainment of local vacuum. This is the general case when the local vacuum entrainment is taken into consideration. To ensure the momentum conservation in this general case, the total charge momentum must be

$$
\boldsymbol{P}_{\boldsymbol{c}}=\frac{U_{e}+U_{m}}{c^{2}}(\boldsymbol{v}+\boldsymbol{u})
$$

where $v$ represent the velocity of charge relative to the local vacuum and $u$ represent the velocity of the local vacuum relative to the distant vacuum.

From (43) can be observed that the total energy is dependent by the gravitational potential, so that the inertial and heavy mass are equals at any velocity and are

$$
m_{i}=m_{h}=\frac{U_{e}+U_{m}}{c^{2}}=\frac{Z_{0}^{2} q^{2}}{8 \pi \alpha} \cdot \frac{1}{\delta}
$$


In the case of an electromagnetic wave, the wave fields form a selfbalanced system, the Poincare stress is zero, the mass is zero (lack of inertia), the total momentum density relative to local and distant vacuum are equals, being given only by the field momentum density

$$
\boldsymbol{p}_{\text {wave }}=\frac{\boldsymbol{E} \times \boldsymbol{H}}{c^{2}}
$$

This may indicate a possible relation between the gravitational effects of the heavy mass and the Poincare stress of it.

\subsection{Mass Dilation}

Let consider the movement through vacuum in the general case, considering a charged particle like in 2.1 moving with constant velocity $v$ through vacuum. We consider the case of low acceleration such that the effects of the acceleration component of the field is negligible compared with the velocity component. The total momentum of moving charge (45) will be

$$
\boldsymbol{P}_{\boldsymbol{c}}=\frac{m_{0}}{\delta}(\boldsymbol{v}+\boldsymbol{u})
$$

Different inertial mass, transversal and longitudinal on the movement direction, are associated with the force of inertia, for

$$
\boldsymbol{a}=\frac{\mathrm{d}}{\mathrm{d} t}(\boldsymbol{v}+\boldsymbol{u})
$$

transversal mass when the acceleration is perpendicular on the movement direction

$$
m_{\perp}=\frac{P_{t o t}}{v+u}=\frac{m_{0}}{\delta}
$$

longitudinal mass when the acceleration is parallel with the movement direction

$$
m_{\|}=\frac{\mathrm{d} P_{t o t}}{\mathrm{~d}(v+u)}=\frac{m_{0}}{\delta^{3}}\left(1+\frac{v u}{c^{2}}\right)
$$

this show the mass dilation but only with velocity $v$ relative to the local vacuum. 


\subsection{Length Contraction}

Now we can extend analysis to the atomic radius to prove that all bodies composed from atoms suffer longitudinal contraction when move through vacuum. First let express the electric field of nucleus, transversal on the direction of atom motion, at $\sin \theta=1$

$$
E_{\perp}=\frac{\mathbb{Z} e}{4 \pi \varepsilon_{0} r^{2}} \cdot \frac{1}{\delta}
$$

this field produce an attractive force over the electron in an orbital position with the centripetal acceleration transversal on the atom motion direction, so in the centrifugal force appear the transversal mass of the electron. Also the presence of a transversal magnetic field of the nucleus due to the atom motion produce an additional repulsive Lorentz force on the electron. This transversal magnetic field is

$$
B_{\perp}=\frac{\mathbb{Z} e}{4 \pi \varepsilon_{0} r^{2}} \cdot \frac{v}{c^{2}} \cdot \frac{1}{\delta}
$$

the centripetal force that act on the electron become

$$
F_{e \perp}-F_{m \perp}=\frac{\mathbb{Z} e^{2}}{4 \pi \varepsilon_{0} r^{2}} \cdot \frac{1}{\delta}\left(1-\frac{v^{2}}{c^{2}}\right)=\frac{\mathbb{Z} e^{2}}{4 \pi \varepsilon_{0} r^{2}} \cdot \delta
$$

Using this and the transversal mass of the electron, result for the transversal orbital radius

$$
r_{n \perp}=\frac{4 \pi \varepsilon_{0} \hbar^{2} n^{2}}{\mathbb{Z} e^{2} \delta m_{e \perp}}=\frac{4 \pi \varepsilon_{0} \hbar^{2} n^{2}}{\mathbb{Z} e^{2} m_{e 0}}
$$

which is the same as the orbital radius at rest. Result that the transversal dimensions of the moving atom and the body, remain unmodified $l_{\perp}=l_{0}$.

Now let make the same analysis longitudinal on the direction of atom motion, at $\sin \theta=0$. No additional magnetic field exist on this direction due to atom motion, the longitudinal electric field of nucleus is

$$
E_{\|}=\frac{\mathbb{Z} e}{4 \pi \varepsilon_{0} r^{2}} \cdot \delta^{2}
$$

the centripetal force that act on the electron is $F_{e \|}=e E_{\|}$, using this and the longitudinal mass of the electron, the longitudinal orbital radius become

$$
r_{n \|}=\frac{4 \pi \varepsilon_{0} \hbar^{2} n^{2}}{\mathbb{Z} e^{2} \delta^{2} m_{e \|}}=\frac{4 \pi \varepsilon_{0} \hbar^{2} n^{2}}{\mathbb{Z} e^{2} m_{e 0}} \cdot \delta
$$


which is shorter than the orbital radius at rest by $\delta$ times. Result that the longitudinal dimensions of the moving atom and the body, in the motion direction, become

$$
l_{\|}=l_{0} \delta
$$

known as Lorentz-FitzGerald contraction or length contraction. Consequently the length contraction is a real phenomenon produced by the movement through vacuum which affect the equilibrium of forces in atom, acting over the atoms and bodies not over the space which is an imaginary concept.

\subsection{Clocks Slowing}

Now we can prove that all clocks based on electromagnetic phenomena become slower, measuring larger time intervals when are in movement through vacuum. The most simple example is that of a light clock, which uses onward and backward propagation time of a light pulse (or electromagnetic field in general) as time base. When this clock is at rest in vacuum its time base will be

$$
\Delta t_{0}=\frac{2 l_{0}}{c}
$$

where $l_{0}$ is the length at rest of the clock arm where the light propagate. If the clock move trough vacuum we have two cases: when the clock arm is transversal on the movement direction and when the arm is parallel with the movement direction. When the arm is transversal the light are forced to propagate over a larger distance through vacuum due to clock movement, the time base become

$$
\Delta t_{\perp}=\frac{2 l_{0}}{\sqrt{c^{2}-v^{2}}}=\frac{\Delta t_{0}}{\delta}
$$

When the arm is parallel the light propagate onward and backward through the moving vacuum over a contracted arm length, the time base become

$$
\Delta t_{\|}=\frac{2 c l_{0} \delta}{(c-v)(c+v)}=\frac{\Delta t_{0}}{\delta}
$$

The results indicate that regardless of clock arm orientation, the time base become larger and the clock slow down in the same way when subjected to movement through the local vacuum. As in the case of length contraction, the internal processes on which the clock operation is based are slowed down, not the time itself which is an imaginary concept. 
All these propagation effects are produced only when exist a movement relative to the local vacuum. If the local vacuum is entrained with the movement of the body, then these effects are reduced down to zero if the entrainment is complete, like in the case of earth or any object on the earth surface.

From (58) in condition of (20) and (22) result that the electromagnetic clocks also slow down when the gravitational potential decrease in the proximity of heavy masses, because will decrease the propagation speed of electromagnetic field and will increase the dimensions of the clock arm. From (22) we can express the arm length

$$
l_{0}=N \cdot r_{n}=N \lambda_{n} \cdot \frac{1}{\sqrt{\Gamma_{0}}}
$$

then we can write for the time base in a lower gravitational potential

$$
\Delta t=\frac{2 l}{c}=2 N \lambda_{n} \cdot \frac{1}{\Gamma}=\Delta t_{0} \cdot \frac{\Gamma_{0}}{\Gamma}
$$

where $\Delta t_{0}$ is the time base where the potential is $\Gamma_{0}$.

\section{The Interpretation of Phenomena}

Some effects and experiments will be analyzed in the new context.

\subsection{The Variation of Vacuum Properties}

The speed of light, the electric permittivity and the magnetic permeability of the vacuum are no longer universal constants, but are dependent by the gravitational potential as shown in equation $(18,19,20)$. This lead to a series of effects like the gravitational refraction of electromagnetic waves when they pass close enough to a massive object. The speed of light decrease gradually toward the center of mass due to the permittivity and permeability increase and this make the light to refract following a curved path around the mass. The effect is very small around masses like sun and earth due to the very high value of the background gravitational potential.

Another effect produced by the vacuum properties variations is the slowing down of electromagnetic processes and clocks in lower gravitational potentials. This in turn will produce the gravitational red shift of light emitted 
from a source localized in a lower gravitational potential if that light is received in an area with higher gravitational potential.

\subsection{Gravitational Entrainment}

In normal conditions the vacuum at the earth surface is almost completely entrained with the orbital movement of earth, because the earth has the dominant gravitational influence on its surface, but is not entrained with the earth rotation around its own axis because this rotation not change equipotential surfaces around the earth. The Michelson-Morley experiment and other experiments like it, give a null result because the vacuum is entrained with the gravitational equipotential lines around the earth, even if the experimental device of this kind move relative to earth, the result is also null due to the length contraction of apparatus and the slowed down processes. The Trouton-Noble experiment with suspended charged capacitor also give a null result for the same reason.

In Sagnac type of experiments the movement of apparatus relative to vacuum is detected. The Sagnac effect appear because the rotation of apparatus relative to earth and vacuum combined with the fact that the light propagate with speed $c$ relative to vacuum, make the two beams of light to propagate over different distances until they reach the receiver. The same effect appear in the case of Michelson-Gale-Pearson experiment where the device rotate with the earth, because the vacuum is not entrained by the earth rotation.

\subsection{The Aberration of Light}

The aberration of light is produced because the light receiver is immersed in the earth vacuum and move with it, the angular deviation of the light beam when enter in the earth vacuum is just an electromagnetic effect (figure 1). The transition from the solar system vacuum (zone 1) to the earth vacuum (zone 2) is gradual with the increasing of the gravitational influence of earth, however for simplicity a step transition is considered instead, the earth vacuum moving with the earth velocity $u$ relative to the solar system vacuum.

The angular deviation is produced because the electromagnetic field is advected by the earth vacuum into the new movement state. The vacuum parameters in both zones are the same, only the movement state is different. Due to advection an additional $\boldsymbol{u} \times \boldsymbol{B}$ (in the case from figure 1 where for 


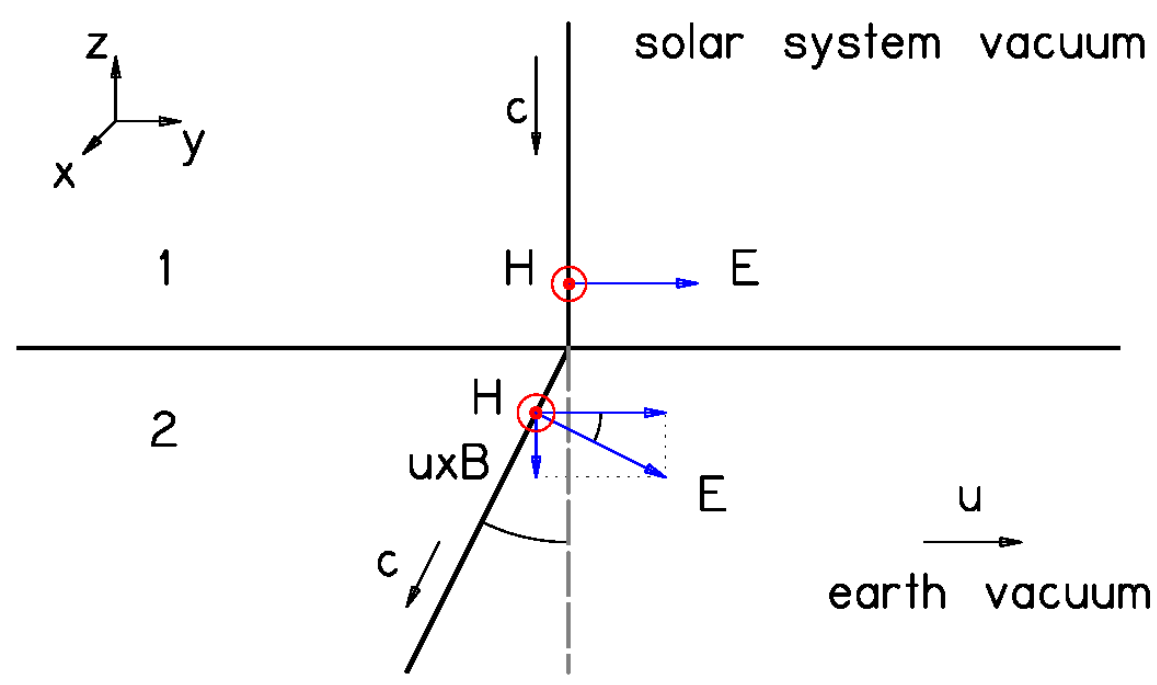

Figure 1: Star light aberration

simplicity the electric field component was chosen parallel with the movement direction, if the magnetic field is chose to be parallel with the movement direction then a $\boldsymbol{D} \times \boldsymbol{u}$ component will be induced) component of electric field is induced in the earth vacuum. The wave magnetic field from the zone 1 being transversal with the direction of movement will pass unaffected in the zone 2 .

$$
H_{1}=H_{2}=H
$$

An electric field component in $-z$ direction is induced in the zone 2 by the passing magnetic component from the zone 1.

$$
E_{2 z}=u \mu_{0} H
$$

In both zones we have the relation

$$
\frac{E}{H}=Z_{0}=\sqrt{\frac{\mu_{0}}{\varepsilon_{0}}}
$$

The angle of aberration is

$$
\sin \vartheta=\frac{E_{2 z}}{E}=\frac{u \mu_{0} H}{E}=\frac{u \mu_{0}}{Z_{0}}=\frac{u}{c}
$$


in the direction opposed to vacuum movement. While the light propagate in that direction is also advected with the vacuum and at any moment the light is located in the extension of zone 1 direction of propagation (dashed line). However an observer located in the zone 2 and moving with the local vacuum will see the light coming at an angle. If we presume that the zone 2 is just a region of moving vacuum between two zone 1 vacuum, an observer located in the zone 1 beyond the moving region of vacuum, will see the light at an unmodified angle and position from original. The same is true if a mirror in the zone 2 reflect the light back to the zone 1, only the travel time of light appear longer, the position remain unmodified. This mean that various regions of vacuum that may move with various velocities between the earth and a star, will not affect the direction and position of the starlight due to their movement, only the movement of earth will have a visible effect.

Also if the light source is moving transversal to its local vacuum, the emitted light is deflected in the direction of source movement (source aberration), however if the source have a spherical emission, then always will exist a beam of light emitted in every direction if the speed of the source is much smaller than that of light. This case become more clear if we consider just a small jerk of the position of star. In consequence only the movement of the receiver and its local vacuum, like an observer on earth, will have an effect on the angle of aberration. This independence of the aberration angle by the movement of spherical light sources was actually observed in the case of binary stars.

In the case of Special Relativity, when the frame of reference is chosen on receiver (earth), the space-time and the electromagnetic field is at rest relative to receiver, and we have only the case of source aberration. But in the case of a source with a spherical front of emission, no aberration angle will be observable for any relative movement between receiver and source. Special Relativity is unable to correctly explain the aberration of light when the frame is chosen on receiver.

\subsection{Fizeau Experiment}

Fizeau water tube experiment, in this experiment the mass of the moving water is too small to have any influence over the vacuum at earth surface, so the vacuum is immobile relative to apparatus which is immobile relative to earth. The moving water which is a dielectric will influence the propagation speed of electromagnetic wave (figure 2). 


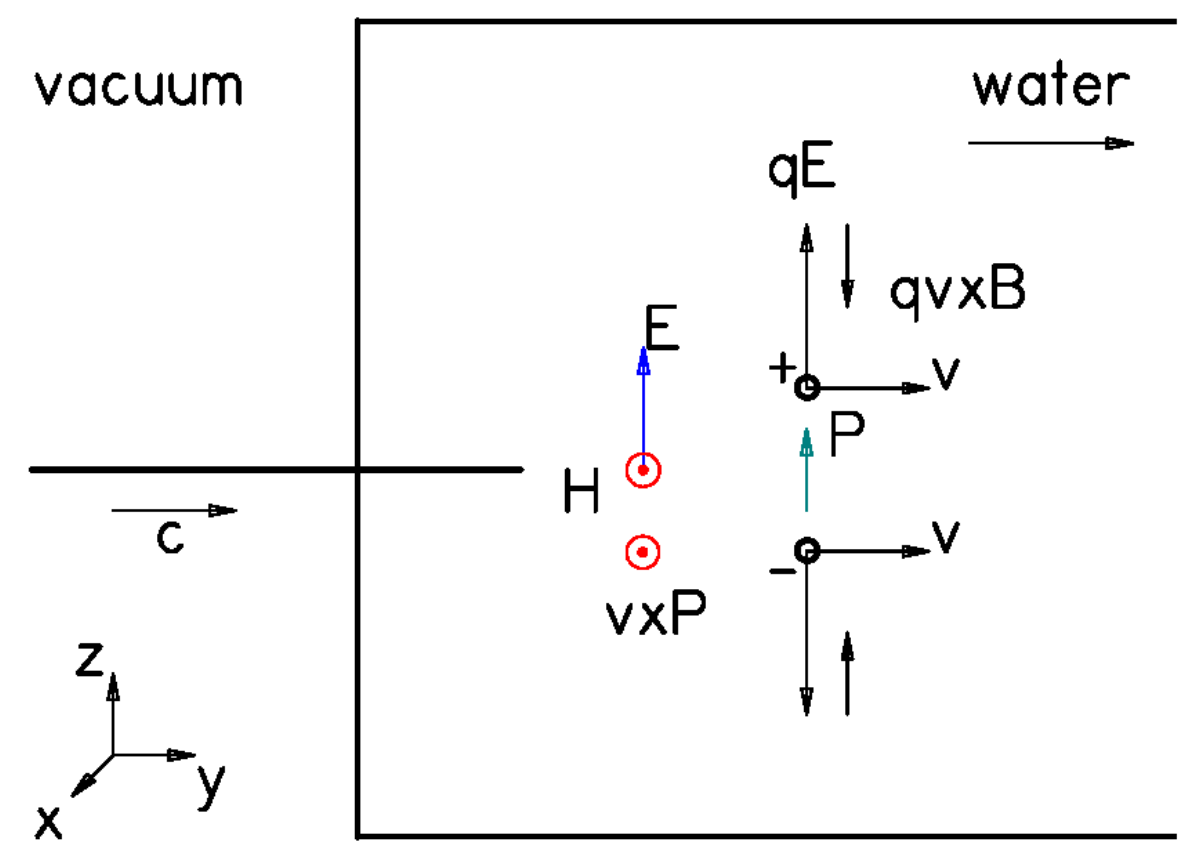

Figure 2: Fizeau water tube experiment

Water being a dielectric the electric field of the wave produce dipoles in water, dipoles which move with the water with velocity $v$ in the direction of wave propagation, in the case illustrated in figure 2. This movement being relative to vacuum produce in turn three effects. First an additional Lorentz forces act over the charges that form the dipole, in the presence of the wave magnetic field. These forces oppose to electric forces if the water move in the direction of wave propagation (like in figure 2), or assist the electric forces if the water move against the direction of wave propagation. Second and the most important, the dipole moving relative to vacuum induce an additional magnetic field which assist or oppose the magnetic field of the wave, depending by water direction of movement in relation with the wave direction of propagation. More precisely the term $\boldsymbol{D} \times \boldsymbol{u}$ from induction equation (33), because $u=-v$, become $\boldsymbol{v} \times \boldsymbol{P}$, the $\varepsilon_{0} \boldsymbol{E}$ term from the expression of electric displacement being immobile relative to vacuum. And third this additional magnetic field induced by the moving dipole also produce 
an additional Lorentz force over the charges that always oppose to electric forces.

We will use the following notations: $\varepsilon=\varepsilon_{0} \varepsilon_{r}, \mu=\mu_{0}, Z, c_{n}$ electromagnetic properties of water at rest; $\varepsilon_{v}, \mu_{v}, Z_{v}, c_{v}$ electromagnetic properties of water in movement. The propagation speed of electromagnetic field is relative to vacuum and apparatus. In the water at rest we have the polarization

$$
\boldsymbol{P}=\varepsilon_{0}\left(\varepsilon_{r}-1\right) \boldsymbol{E}
$$

in this case we also have

$$
\frac{E}{H}=Z
$$

when the water move in the particular case from figure 2, we have

$$
\begin{gathered}
\boldsymbol{P}=\varepsilon_{0}\left(\varepsilon_{r}-1\right)(\boldsymbol{E}+\boldsymbol{v} \times \boldsymbol{B})=\varepsilon_{0}\left(\varepsilon_{r}-1\right)[E-v \mu(H+v P)] \mathbf{k} \\
P\left[1+\left(\varepsilon_{r}-1\right) \frac{v^{2}}{c^{2}}\right]=\varepsilon_{0}\left(\varepsilon_{r}-1\right)(E-v \mu H)
\end{gathered}
$$

if we note

$$
\xi=1+\left(\varepsilon_{r}-1\right) \frac{v^{2}}{c^{2}}
$$

we have

$$
P=\frac{1}{\xi} \varepsilon_{0}\left(\varepsilon_{r}-1\right)\left(E-\frac{v \mu E}{Z}\right)=\frac{1}{\xi} \varepsilon_{0}\left(\varepsilon_{r}-1\right)\left(1-\frac{v}{c_{n}}\right) E
$$

the electric displacement become

$$
D=E\left[\varepsilon_{0}+\frac{1}{\xi} \varepsilon_{0}\left(\varepsilon_{r}-1\right)\left(1-\frac{v}{c_{n}}\right)\right]=\varepsilon_{v} E
$$

The moving water impedance is

$$
Z_{v}=\frac{E}{H+v P}=\frac{Z}{1+\frac{v}{\xi} Z \varepsilon_{0}\left(\varepsilon_{r}-1\right)\left(1-\frac{v}{c_{n}}\right)}
$$

The speed of light in the moving water relative to vacuum is

$$
c_{v}=\frac{1}{\varepsilon_{v} Z_{v}}=\frac{\xi+v Z \varepsilon_{0}\left(\varepsilon_{r}-1\right)\left(1-\frac{v}{c_{n}}\right)}{\xi Z \varepsilon_{0}+Z \varepsilon_{0}\left(\varepsilon_{r}-1\right)\left(1-\frac{v}{c_{n}}\right)}
$$


Considering the equalities

$$
\begin{gathered}
\varepsilon_{r}=n^{2} \\
c_{n}=\frac{c}{n} \\
Z \varepsilon_{0} \varepsilon_{r}=\frac{1}{c_{n}}
\end{gathered}
$$

after some calculations result

$$
c_{v}=\frac{\xi c_{n}+v \cdot \frac{\varepsilon_{r}-1}{\varepsilon_{r}}\left(1-\frac{v}{c_{n}}\right)}{1-\frac{\varepsilon_{r}-1}{\varepsilon_{r}}\left(\frac{v}{c_{n}}-\frac{v^{2}}{c^{2}}\right)}
$$

and finally

$$
c_{v}=\frac{\xi \frac{c}{n}+v\left(1-\frac{1}{n^{2}}\right)\left(1-\frac{n v}{c}\right)}{1-\left(1-\frac{1}{n^{2}}\right)\left(\frac{n v}{c}-\frac{v^{2}}{c^{2}}\right)}
$$

which is the propagation speed of light when the water move in the direction of propagation. Because in the case of Fizeau experiment the water velocity was much smaller than $c$, the propagation speed can be approximate as

$$
c_{v 0}=\frac{c}{n}+v\left(1-\frac{1}{n^{2}}\right)
$$

which is the Fresnel formula confirmed by Fizeau in his water tube experiment. In equations (64 and 65) the water velocity $v$ become $-v$ in the case of a changed water flow.

\section{Conclusion}

If we take into consideration that the vacuum background is intimately connected with the gravity, then we can explain the electrodynamics and gravitational phenomena with simple Galilean invariance, which preserve causality and allow us to correctly understand these phenomena. In fact the gravity 
and the vacuum background represent the same thing, the observable gravitational effects are only the effects of properties variation in the vacuum background. The vacuum background becomes the preferred frame of reference, but this frame is not unique and absolute in the entire universe, nor arbitrary relative, instead is dependent in every point by the distribution and movement of matter.

\section{References}

[1] C.I. Mocanu, Teoria Campului Electromagnetic (Theory of Electromagnetic Field), 1981, Ed. Didactica si Pedagogica

[2] Bo Thide, Electromagnetic Field Theory, ISBN 978-0-486-4773-2

[3] Albert Einstein, Sidelights on Relativity, 1922, translated by J.B. Jeffery and W. Perrett

[4] Oliver Heaviside, Electromagnetic Theory, vol. 1-2-3

[5] Kirk T. McDonald, Electromagnetic Field Momentum, Joseph Henry Laboratories, Princeton University, Princeton, NJ 08544 (Aug. 30, 1995; updated June 9, 2012)

[6] Cornelia Motoc, Fizica (classic and quantum physics) vol. 1+2, 1994, Ed. All

[7] J.C. Maxwell, A Treatise on Electricity and Magnetism, vol. 1-2

[8] L. Essen, The Special Theory of Relativity A Critical Analysis

[9] Tom Van Flandern, The Speed of Gravity - What the Experiments Say, Physics Letters A, 250 (1998) 1-11 